\title{
In Vitro Models of the Liver: Disease Modeling, Drug Discovery and Clinical Applications
}

\author{
Scott D. Collins ${ }^{1,2} \bullet$ Gloria Yuen ${ }^{1,2} \bullet$ Thomas Tu ${ }^{3,4} \bullet$ Magdalena A. \\ Budzinska1,5,6 • Kevin Spring ${ }^{5,6} \bullet$ Katherine Bryant ${ }^{1,2} \bullet$ Nicholas A. Shackel $^{1,2}$ \\ ${ }^{1}$ Gastoenterology and Liver Laboratory, Ingham Institute for Applied Medical Research, \\ Liverpool, New South Wales, Australia; ${ }^{2}$ South Western Sydney Clinical School, University \\ of New South Wales, Sydney, Australia; ${ }^{3}$ Department of Molecular Virology, University \\ Hospital Heidelberg, Heidelberg, Germany; ${ }^{4}$ German Center for Infection Research (DZIF), \\ Heidelberg Partner Site, Heidelberg, Germany; ${ }^{5}$ Medical Oncology Group, Ingham Institute \\ for Applied Medical Research, Liverpool, Australia; ${ }^{6}$ Liverpool Clinical School, Western \\ Sydney University, Liverpool, Australia
}

Author for Correspondence: Nicholas Shackel, South Western Sydney Clinical School, University of New South Wales, Sydney, Australia. Email: n.shackel@unsw.edu.au Doi: http://dx.doi.org/10.15586/hepatocellularcarcinoma.2019.ch3

\begin{abstract}
In vitro models of the liver have led to important insights into the pathogenesis of liver disease. These models are essential tools in the discovery and preclinical stages of drug development. The clinical application of these models is also emerging as a promising avenue for validating genetic target-matched treatments, in a precision medicine approach to treatment. Recent advances in 'liver-on-a-chip' technology and liver organoid research have opened up new opportunities for the functional and clinical use of organotypic in vitro models. This chapter focuses on the currently available in vitro liver models and the opportunities and limitations they present in the context of evaluating their use in disease modeling, drug discovery, and clinical application.
\end{abstract}

Keywords: liver-on-a-chip; organotypic cultures; precision-cut tissue slices; sandwich-cultured hepatocytes; whole organ explants.

In: Hepatocellular Carcinoma. Janina E.E. Tirnitz-Parker (Editor), Codon Publications, Brisbane, Australia. ISBN: 978-0-9944381-8-8. 2019; Doi: http://dx.doi.org/10.15586/ hepatocellularcarcinoma.2019

Copyright: The Authors.

License: This open access article is licensed under Creative Commons Attribution 4.0 International (CC BY 4.0). https://creativecommons.org/licenses/by-nc/4.0/ 


\section{INTRODUCTION}

The large burden of liver disease and primary liver cancer along with the management difficulties encountered have provided the impetus to pursue the use of representative in vitro models of liver function, response to injury, and development of malignancy. Improved 2D and 3D in vitro disease models would enhance our understanding of the cause of liver injury and cancer, increase the efficacy of preclinical drug discovery, and be a useful clinical tool for precision medicine. The increasing popularity of organ-on-a-chip technology and improvements in 3D cell cultures has enabled unique insights into liver disease $(1,2)$. This chapter focuses on the current types of in vitro liver models, the opportunities and limitations of their uses in drug discovery, basic research and clinical management, as well as new directions of this field.

\section{IN VITRO LIVER MODELS}

In this overview, in vitro models will be defined as the culturing of isolated tissue components of an organ, while preserving many aspects of the in vivo environment. The chief purpose of in vitro models in research and medicine is to minimalize experimental variables to effectively isolate different organ components or structures for study under well-controlled, reproducible, and easily assessed conditions. This overview will focus on 2D and 3D models of the liver based on organotypic characteristics, including cell type, liver function, and zonation, and likely application in basic research, drug discovery, and clinical practice (Figure 1).

The liver has a heterogeneous cellular composition that includes hepatocytes (the target of most disease and comprise the majority of the liver in quantity and volume), Kupffer cells (liver-resident macrophages), hepatic stellate cells, liver sinusoidal endothelial cells (LSECs), biliary epithelial cells, fibroblasts, immune cells, and adult stem cells. Important liver functions to consider include: (i) the metabolism of endogenous substrates and exogenous compounds; (ii) the regulation of amino acids, carbohydrates, and fatty acids; (iii) synthesis of proteins (such as albumin or transferrin) and bile synthesis; (iv) immune activation upon injury; (v) the biotransformation of xenobiotics; and (v) the resilience to senescence $(1,2)$. The cytochrome P450 (CYP450) family of abundant enzymes is also significantly important to liver function as they mediate the metabolism of drugs (3).

The lobules of the liver are complex with perivenous, intermediary, and periportal zones (4). The intercellular oxygen concentration of the lobule is $15-20 \mathrm{~mm}$ $\mathrm{Hg}$ in the perivenous zone compared to $45-50 \mathrm{~mm} \mathrm{Hg}$ in the periportal zone (5). Metabolic processes, including glucose uptake, glycolysis, amino acid synthesis, bile acid production, and glucuronidation, are all greater in perivenous cells, which also have the greater CYP450 enzyme activity. By contrast, oxygen uptake, glucose delivery, gluconeogenesis, urea synthesis, fatty acid oxidation, cholesterol synthesis, and sulfation are all comparatively greater in periportal cells. Non-parenchymal cells such as bile duct cells and hepatic stellate cells are more abundant in the oxygen-rich periportal zone. These observations are important to liver modeling 


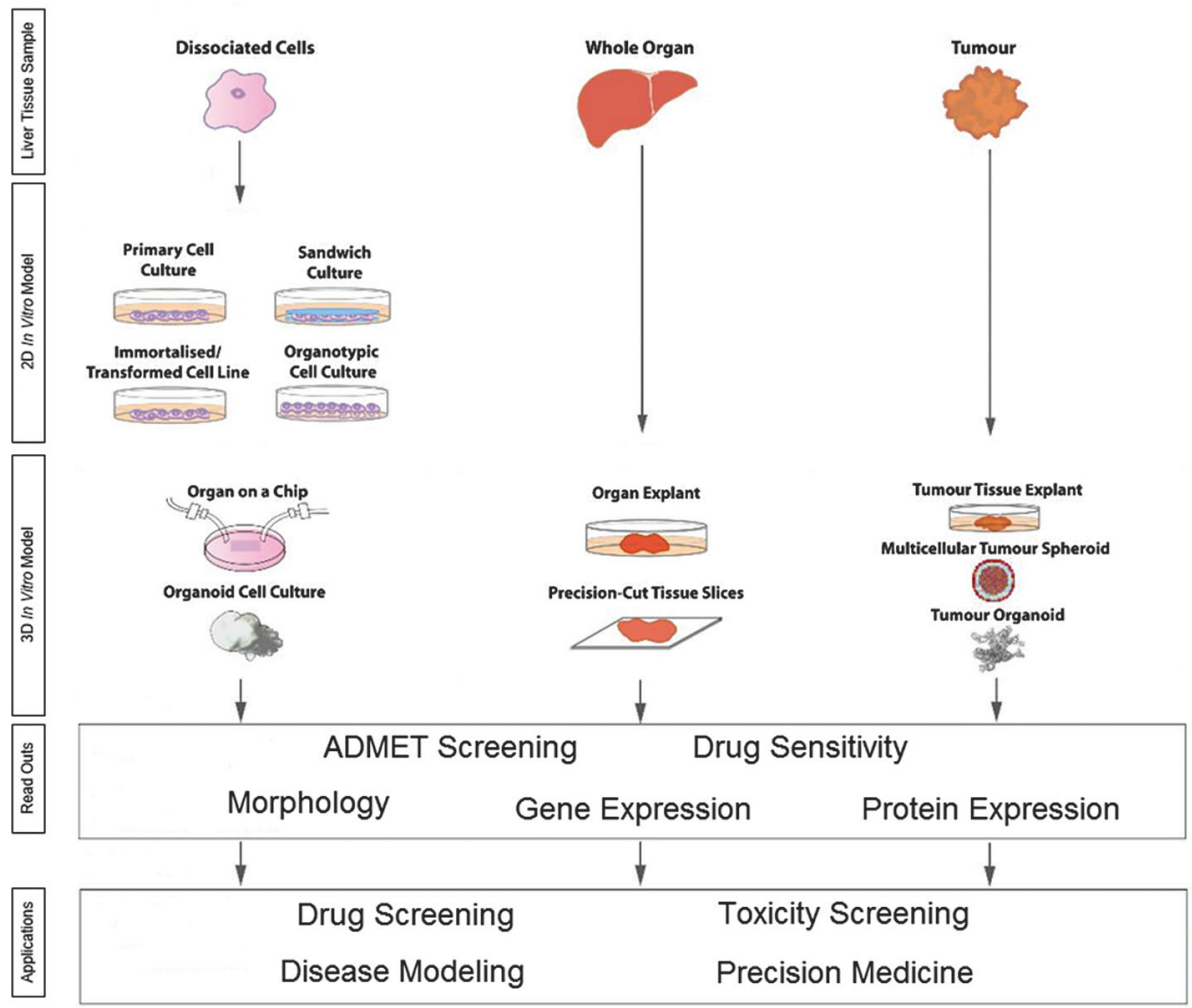

Figure 1 Overview of 2D and 3D in vitro models of the liver. Flow diagram indicates the in vitro models of the liver, their readouts, and applications. Each model was categorized by the type of sample it is derived from and whether it is 2D or 3D model.

as zonation is important and disrupted in liver diseases, especially diseases associated with hypoxia and reactive oxygen species like non-alcoholic fatty liver disease (NAFLD) and hepatocellular carcinoma (HCC) (6).

Increasing the efficacy of drug development and toxicity testing by improving in vitro models is of great interest to researchers and the pharmaceutical industry (7). In 2015, the cost of bringing a new drug to market was estimated at 2.6 billion USD (8), with the major contributor to this cost being the very low clinical success rate of new compounds (approx. 11.8\%) (9). This high burden of cost necessitates the exploration of new approaches, including advances in preclinical methods, which select new drug interventions for clinical trials.

In the discovery and preclinical development stages of drug development, candidates are identified by correlating drug responses in cell cultures and preclinical animal models_-usually one rodent and one non-rodent species (10). Screening for absorption, distribution, metabolism, excretion, and toxicity (ADMET screening, also commonly referred to as ADME or the study of drug metabolism and pharmacokinetics) optimizes preclinical testing by enabling better understanding of the pharmacokinetic and pharmacodynamic properties of drug candidates (11). 
Desirable drug-like properties identified by ADMET screening include adequate absorption and distribution, low metabolism, complete elimination from the body, and a minimal toxicological risk (10).

A significant challenge in this field is predicting human-specific liver toxicity (12). Animal models do not always reflect human toxicity due to differences in physiology, interspecies metabolic capacities, and disease adaptations. Similarly, in vitro models often do not accurately predict toxicity due to non-linear dosetoxicity relationships, unclear mechanisms, non-organ-specific toxicity, as well as adverse downstream effects $(1,12)$. Drug-induced hepatic injury is the most frequently cited reason for approved drugs being removed from the market (13). Current 2D in vitro assays are based on cell lines such as HepG2 that have reduced metabolic capacities compared to primary hepatocytes, while the use of primary human hepatocytes suffers from high donor-to-donor variation and cultures only retain in vivo characteristics for a short time ex vivo (11). The effect of improving these in vitro assays may potentially lead to more effective and rapid pre-clinical drug development.

After the completion of the human genome project in the early 2000s, there was significant optimism for the potential of genomic medicine to revolutionize the diagnosis and treatment of many illnesses, in particular, the clinical application of genetic predictors to better understand patient risks of disease and responsiveness to potential designer drugs, based on targeting specific molecular pathways (14). In 2011, the US National Research Council coined the term "precision medicine" to inspire a new taxonomy for disease via a knowledge network. They defined precision medicine as

"The tailoring of medical treatment to the individual characteristics of each patient $[\ldots]$ to classify individuals into subpopulations that differ in their susceptibility to a particular disease or their response to a specific treatment. Preventative or therapeutic interventions can then be concentrated on those who will benefit, sparing expense and side effects for those who will not. This is different from personalized medicine, which refers to 'an approach to patients that considers their genetic make-up but with attention to their preferences, beliefs, attitudes, knowledge and social context'." (15)

The disease treatment strategies that have so far benefited the most from precision medicine are treatments for cystic fibrosis and cancer management using genome sequencing to enhance patient care by improved diagnostic sensitivity, allowing for more precise genetic therapeutic targeting (16). Since the early success of the Bcr-Abl kinase inhibitor Imatinib for targeted therapy for chronic myeloid leukemia, oncology has moved towards molecular classification (16), but currently there are only 11 genomic alterations known to drive tumor progression in different tissues matched directly with approved targeted therapies (17).

\section{CONVENTIONAL 2D IN VITRO LIVER CELL CULTURES}

Essentially, cell biology relies on 2D models generated from dissociated cell cultures that are expanded on plastic surfaces, often supported by extracellular matrix (ECM) scaffolding. These are primary cell cultures derived directly from 
harvested tissue or immortalized cell lines (primary cells genetically transformed to produce rapidly proliferating, uniform, easily cultured, artificial phenotypes). A major reason for the popularity of dissociated cell cultures is that the majority of mammalian cells can be expanded into adherent colonies on culture plates, and these have proven to be relatively low cost and easy to manipulate and maintain. A high-throughput cultured monolayer of cells receives a consistently homogenous amount of nutrients, growth factors, and exposure to oxygen. Commercialized cell lines are available across a diverse range of tissue types, and there is extensive commercial support for these cultures, such as the availability of different culture media and consumables. Furthermore, there are various options for genetic manipulation, such as CRISPR, gene transfer, insertion, deletion, silencing, and cell fusion (1).

\section{Primary cell cultures}

Human hepatocyte primary cell cultures are a physiologically relevant model for studying drug biotransformation and toxicity $(18,19)$. However, cells grown in this way have a number of issues. They only maintain their wild-type characteristics for a limited time when cultured on 2D surfaces because of de-differentiation. In addition, in vitro manipulation often results in a loss of wild-type characteristics, slow proliferation, changes in metabolism, and early senescence after a limited number of passages $(18,19)$. Therefore, cell cultures require successive tissue harvests, which incur higher associated costs. Moreover, the harvesting of tissue is susceptible to contamination from non-applicable cell types, thereby compromising the model's integrity (1).

The ECM has a profound effect on primary cell function, differentiation, signaling, and morphology $(20,21)$. For example, culturing primary hepatocytes with the scaffold matrix Matrige ${ }^{\circledR}$ induces gene expression, which more closely resembles liver tissue in vivo. It also improves cellular morphology by enhancing cuboidal shape and results in cells with clearly defined cell borders that allow the formation of highly organized cellular networks (22).

Primary hepatocyte cell cultures have been useful for understanding the mechanisms in liver regeneration (23) and for discerning the relationship between the liver cytoskeleton and liver-specific protein expression (24). Similarly, primary cultures of hepatic stellate cells have been instrumental in understanding the causes of liver fibrosis and identification of key fibrogenic mediators $(25,26)$. In drug testing, primary human hepatocyte cell cultures are considered the "gold standard" because they display many phenotypic functions of the liver when compared to other in vitro models $(27,28)$. However, this approach has been heavily criticized as suboptimal. The common issues include: (i) cells being cultured at densities of only approximately $1 \%$ of physiologically normal tissue densities, thereby impairing intercellular signaling; and (ii) cultures being non-homeostatic as conditions are optimized for rapid growth, thereby preventing correct cell differentiation $(29,30)$. Primary hepatocytes experience a decline in CYP450 expression when grown in vitro (31), while the transcription of common genes is unaffected leading to a decrease in CYP450 proteins and activity, significantly limiting the translatability of this model (32). 


\section{Sandwich-cultured hepatocytes}

Culturing primary hepatocytes between two layers of collagen, termed sandwichcultured hepatocytes $(\mathrm{SCH})$, results in retained cellular polarity with correct localization of basolateral and canalicular transporters as well as formation of functional bile networks $(33,34)$. Discovered by Dunn and colleagues, SCH maintain mRNA expression, as well as cell functions, such as the secretion of albumin, transferrin, fibrinogen, bile acids, and urea for 6 weeks $(35,36)$, and CYP450 isozymes for 2 weeks (37). SCH have proven to be a useful tool to study hepatobiliary drug disposition and mechanisms of drug-induced liver injury, for example, elucidating transport mechanisms responsible for the elimination of the antifungal agent, micafungin (38), and the mechanisms of bile acid-mediated, drug-induced liver injury (39).

\section{Immortalized or transformed cell lines}

Immortalized or transformed cell lines are dissociated cell cultures, which have been genetically modified or selected for an oncogenic phenotype. Typically, these cultures show rapid proliferation, resistance to de-differentiation, improved passaging, and greater resilience to senescence, making these cells convenient to maintain, expand, and retain phenotypic consistency between experiments. These cell cultures have been successfully used to study hepatitis B virus (HBV) and hepatitis D virus (HDV) infections. Mechanisms of HBV viral entry were discovered in HepRG cell lines (40), the expression/replication of HBV was discovered in HepG2 (41), and the complete HDV replication cell cycle was discovered in HepG2 and Huh7 cells $(42,43)$. The shortcomings of these cell lines include significant changes in differentiation potential; altered genomic content (44); abnormal proteome expression; and the loss of features such as cellular polarity (45), contact inhibition $(46,47)$, metabolic CYP450 potential $(48,49)$, the induction of inflammatory mediators (50), as well as paracellular transport (51).

Due to most immortalized human hepatic cell lines having reduced liverspecific functionality (52), different strategies have been used to counteract this issue, including co-culture systems with primary human hepatocytes and overexpressing liver-enriched transcription factors, CYP450 enzymes, or proliferation inhibitors to increase hepatic functions (52). Immortalized human hepatic cell lines have been successfully used to investigate the life cycle of hepatitis $\mathrm{C}$ and $\mathrm{B}$ viruses (53-56), and they act as cellular models of hepatocarcinogenesis (57) and steatosis (58). Furthermore, immortalized hepatic cell lines have also been found suitable as in vitro tools for drug screening and safety testing. Hc3716-hTERT, immortalized fetal hepatocytes, and telomerase-immortalized hepatic stellate cells NPC-hTERT have been used as models for predicting the side effects of telomeretargeting drugs (59), and Fa2N4 cells have been used for screening pregnane $\mathrm{X}$ receptor-mediated CYP3A4 induction (52).

\section{Organotypic cultures}

A major limitation of dissociated cell cultures is their high degree of homogeneity as they fail to represent liver tissue heterogeneity. While hepatocytes comprise the 
majority of cells within the liver, liver function is dependent on a number of different cell types. 2D organotypic culture uses multiple different cell types to recapitulate in vivo-like cell heterogeneity. Co-cultures of hepatocytes and macrophages have been successfully used to model their intercellular cross-talk, their roles in the regulation of liver regeneration, hepatocyte function, and the acute-phase response to septic liver injury (60). Long-term co-cultures of hepatocytes and LSECs, either on top of or sandwiched between a collagen gel, retained the LSEC phenotype and enhanced hepatocyte functions, such as increased CYP450 activity (61). In contrast, co-cultures of primary hepatocytes and endothelial cells, maintained under high oxygen conditions, preserved cell morphology, high CYP450 levels, and native gene expression (62). A recent example by Ware and colleagues was a triculture of primary human hepatocytes with 3T3-J2 fibroblasts and LSECs overlayed with Matrigel ${ }^{\circledR}$, which was shown to display a stable phenotype with increased albumin and urea secretion for 3 weeks (63).

\section{Shortcomings of conventional 2D liver cell cultures}

While these models have many benefits, a significant issue with 2D liver models is their lack of hepatic sinusoid heterogeneity, in vivo-like cell density, oxygeninduced zonation, and the liver circulatory system. The clinical application of 2D cell cultures is limited due to significant issues of cell contamination, nonreflective cell differentiation, genetic drift, variable drug responsiveness, and a limited capacity to predict toxicity, creating a degree of uncertainty when using 2D culture as a model for potential treatments, with a possible exception of patient-derived tumor cell lines for precision medicine (64).

\section{D IN VITRO LIVER MODELS}

The shortcomings of 2D cell culture models have driven the development of 3Dcell culture techniques. The advantages of 3D models include replicating the complex attributes of the liver beyond liver-specific metabolism, such as increased cell density, organization, and cell-cell signaling, $\mathrm{O}_{2}$ zonation, as well as the anatomy of the liver lobule and the circulatory system (Figure 2). Some of these models are limited by their low applicability for high-throughput screening as well as their laborious preparation, lack of reliable protocols, and short-term survival of these models in culture. However, 3D models have proven useful in developmental and toxicological studies and represent an exciting opportunity for more functionally relevant clinical modeling.

\section{Whole organ explants}

Whole mouse liver organ explants have been used to study the effects of oxidation on the progression of hepatocarcinoma. In 2016, Torricelli and colleagues reported inoculating the murine hepatocarcinoma cell line Hepa 1/Als into the livers of live mice, which proliferated in vivo for 20 days before the livers were removed and used as a whole organ explant model to study the effects of the antioxidant Citozym on tumor size in culture over a 4-week period (65). 


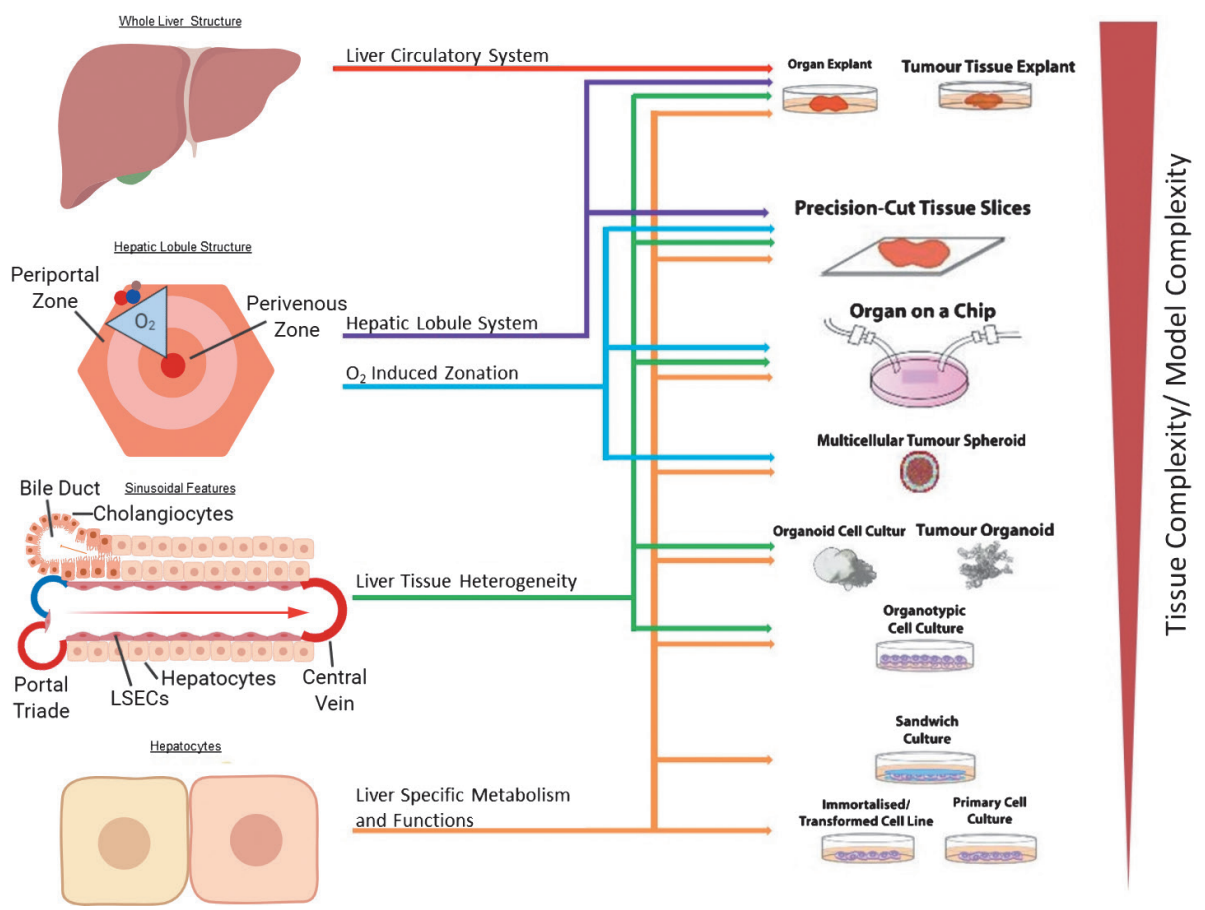

Figure 2 Different levels of structural complexity in the liver and their attributes represented in in vitro models. The different structures of the liver and their corresponding liver models on a gradient, based on their tissue complexity. Structures of the liver are then linked by attributes represented in the in vitro models discussed in this chapter.

\section{Precision-cut tissue slices}

Liver 'precision-cut tissue slices' (PCTS) have mostly been generated using rat livers, but the technique has also been used for other species including humans (66). Slicing allows sufficient oxygen and nutrient supply to the inner cell layers, and hepatocytes retain their membrane and intracellular polarization (67). In a study by Vickers and colleagues, rat liver slices have been found to be fully capable of metabolizing compounds and maintaining fibrogenic pathways, such as activation of stellate cells, the proliferation of myofibroblast-like cells, and an increased collagen deposition for 4 days under appropriate conditions (68). As with 2D cultures, CYP450 expression decreases during prolonged culturing, but this has been shown to slow down when the medium is supplemented with insulin, dexamethasone, and fetal calf serum $(69,66)$.

\section{Tumor tissue explants}

A 'tumor tissue explant' is a 3D model of cancer, where an excised human tumor is embedded in collagen and tissue culture medium (70). Mainly used as an in vitro model of drug efficacy, this method has been demonstrated by Vaira and 
colleagues to preserve pathway activation, pharmacological inhibition, internal 3D architecture, cell viability, and global gene expression profiles up to 5 days ex vivo (71). Unfortunately, this model is relatively unreproducible due to tissue heterogeneity, applicability of techniques such as imaging and flow cytometry is limited, and the culture is only viable for a short period of time, making it impractical for any form of high-throughput, long-term, or clinical investigations (72).

\section{Multicellular tumor spheroid}

The best-characterized 3D organotypic models of cancer are "multicellular tumor spheroids," which are constructed from homogeneous tumor cells or co-cultures on nonadherent surfaces, where the cell suspension undergoes aggregation and compaction $(73,72)$. Spheroids re-establish morphological, functional, and physiological cellular transport properties of their corresponding tissue and resemble the avascular tumor nodules/micrometastases or intervascular regions of large solid tumors (74). These have been used to gain insights into therapeutic challenges associated with drug resistance, metabolic and proliferation gradients, and the importance of cell-cell/cell-matrix interactions (74). Liver multicellular tumor spheroids have been used for understanding microenvironmental chemoresistance of HCC associated with the crosstalk between HCC cells, hepatic stellate cells and other stromal cells $(75,76)$. For instance, liver cancer spheroids of Huh7 cells co-cultured with human umbilical vein endothelial cells promoted HCC gene expression and oncogenic properties, such as cell proliferation, increased expression of cancer stem cell markers, and extracellular cytokine-mediated signaling (77). Furthermore, this multicellular tumor spheroid model tolerated higher anti-cancer drug concentrations than the monolayer control, which may be due to the hypoxic conditions within the spheroid, activating extracellular signalregulated kinases (ERK), critical in tumor cell proliferation (77).

\section{Organ-on-a-chip}

An "organ-on-a-chip" utilizes the microfluidic technology to replicate the in vivo microenvironment and homeostasis of living human organs (78). Often consisting of transparent 3D polymeric microchannels lined by human tissue cultures, these devices are designed to mimic the 3D microarchitecture, organ-specific mechanical/ biochemical microenvironment, and the functional tissue-tissue interfaces in organs. Many investigators use micro-channels of matrix-coated porous membranes with a layer of endothelial cells, populated by the desired co-culture, connected by wells containing the preferred perfusion medium (78). These devices have also been designed with compartmentalized channels, allowing for independent fluidic/aerosol access to individual tissue types, enabling selective treatment conditions and analysis (73). "Liver-on-a-chip" systems have been shown to predict clearances, toxicity, and the mechanism of action of certain drugs (79).

Commercially available liver-on-a-chip microfluidic systems such as the 3D perfused cell culture platform from Zyoxel and the Microliver chip from $\mathrm{H}_{\mathrm{Re}}{ }^{\circledR}$ corporation have been used for toxicity testing, but none of these systems have been fully validated. Most current models use primary human hepatocytes to populate the system, and a few include a co-culture with non-parenchymal cells, 
which has improved their capacity to predict liver toxicity (80). For example, the anticancer prodrug Flutamide was tested for hepatotoxicity using human HepG2/ C3a cells in a microfluidic biochip and led to metabolic results consistent with reports in the literature. The authors demonstrated perturbation of the tricarboxylic acid cycle and impaired urea cycle with reduced uptake of essential amino acids (81). In 2016, Bhise and colleagues have also had success in drug toxicity analysis with a liver-on-a-chip platform using human HepG2/C3a spheroids encased in hydrogel within a bioreactor for long-term culturing (82). Furthermore, biochips using primary hepatocytes have been used to measure the pharmacokinetics of several drugs, with results that resemble data in relevant clinical trials (83). The use of human-induced pluripotent stem cells (hiPSCs) to generate hepatocyte-like cells has been assessed for populating liver-on-a-chip systems. However, differentiated cells were found to have reduced functionality and immature gene/protein expression (84). Focused efforts at recapitulating lobule zonation using liver-on-a-chip systems have had mixed success $(85,86)$. A controlled oxygen gradient has been maintained in primary rat hepatocytes, which induced in vivo-like heterogeneous CYP450 localization and toxicity. This is significant because most studies only model one lobule zone (usually the perivenous zone), and hence, the expression of intermediate metabolites may be exaggerated, while detoxification may be underestimated (79).

\section{Organoids}

An "organoid cell culture" is defined as a collection of several cell types that develop from stem cells or organ progenitors, display organ-specific functions, mimic its structural organization, and self-organize through cell sorting and spatially restricted lineage commitment, similar to organogenesis in vivo (87). Organoids are usually formed by exploiting the expansion potential of three cell types: pluripotent embryonic stem (ES) cells, induced pluripotent stem cells (iPSC), or organ-specific adult stem cells (aSC), forming new primary tissue buds, made of self-organizing daughter cells that are induced to differentiate in culture. These daughter cells display the capacity to self-organize into structures that reflect crucial aspects of the tissue for which they are fated (88). What distinguishes 3D liver organoid cell cultures from other in vitro models is that they bridge the gap between the microenvironmental integrity of organ explants and PCTS, yet have the high-throughput accessibility of immortal cell lines.

Liver organoids have demonstrated advantages over conventional in vitro models such as long-term genetic stability, in vivo-like organization, and maintaining the necessary cellular crosstalk and behavioral characteristics of their primary corresponding cells (89). For example, adult stem cells from alpha-1 antitrypsin (A1AT)-deficient patients cultured into liver organoids mimic the in vivo situation with AlAT protein aggregates and signs of endoplasmic reticulum stress (89). Liver organoids were first created by Huch and colleagues by exploiting the expansion potential of $\mathrm{LGR}^{+}$progenitor/stem cells in damaged adult mouse liver tissue, by Wnt-driven regeneration. They then induced hepatocyte maturation by inhibiting Notch and TGF- $\beta$ signaling, which led to the expression of genes involved in cholesterol and lipid metabolism, as well as from the CYP450 superfamily. Immunofluorescent analysis revealed the expression of hepatocyte 
nuclear factor $4 \alpha$ and albumin, hepatocyte binucleation, as well as patches of the progenitor cell and ductal marker cytokeratin 19. Ninety percent of these cells were also competent for low-density lipoprotein uptake and accumulated glycogen (90).

In addition, Huch and colleagues established the first organoid culture system for human liver from primary bile duct stem cells (89). These organoids displayed high stability, both chromosomally and structurally, with low rates of genetic alterations over a 3-month culture. Using the established methods developed for mouse liver organoids, they induced hepatocyte differentiation in the human liver organoids. As a consequence, the cultures began to display hepatocyte gene expression, albumin secretion, CYP450 metabolism, bile acid production, ammonia elimination, low-density lipoprotein uptake, and glycogen storage (89). Further, organoids were readily engrafted in vivo upon transplantation in mice (89).

It has been proposed that liver organoids may be a useful model for studying the transition of NAFLD to non-alcoholic steatohepatitis if these organoids were co-cultured with hepatic stellate cells, Kupffer cells, and other inflammatory cells (91). Retroviral transduction and liposomal transfection have been successfully used to genetically manipulate liver organoids with green florescent proteinexpressing vectors (92). Another exciting avenue to explore is CRISPR gene editing, with success already achieved using intestinal organoids of cystic fibrosis patients, where the cystic fibrosis transmembrane conductance regulator (CFTR) locus was corrected in vitro by homologous recombination (93).

Although 3D liver organoid cell cultures are becoming a research focus, challenges for the technology include the recapitulation of the in vivo ECM. It has been suggested that the use of decellularized liver ECM populated with liver organoids may improve hepatocyte functions (89), which has had success in promoting survival and maturation compared to collagen type I (94). Limitations of liver organoids include the lack of a native microenvironment, thus inhibiting the study of the interactions between stem cells and their niches, a lack of all necessary in vivo growth factors or signaling gradients, and an inability to accurately model immune responses. A possible solution to this is organotypical co-culturing and the application of microfluidic technologies. Further heterogeneity between organoid cultures can cause inconsistency in reproducing phenotypic traits such as size, shape, cellular composition, and 3D architecture $(95,96)$.

In drug development, an in vitro organoid system comprised of human cells which are complex enough to demonstrate organotypic composition, morphology, and functionality (Table 1) would be ideal in closing the gap in phenotypic drug discovery (26). Increasing the chain of translatability for target-agnostic investigations remains a significant challenge (3), and human organoids may build a rational and sustainable discovery pipeline, reducing false-positives and cost. The reason for this is that organoids may present a more phenotypical disease-associated functional response to treatment than 2D cell lines as well as a more accurate disease-free associated phenotype. Phenotypic drug discovery with generic readouts like viability or apoptosis in cancer cell lines often provides little insight into disease pathways or mechanisms of action, while in vitro 3D organoid models exhibit the potential to become highly predictive cell-based tools for preclinical drug toxicity assessments (97). 


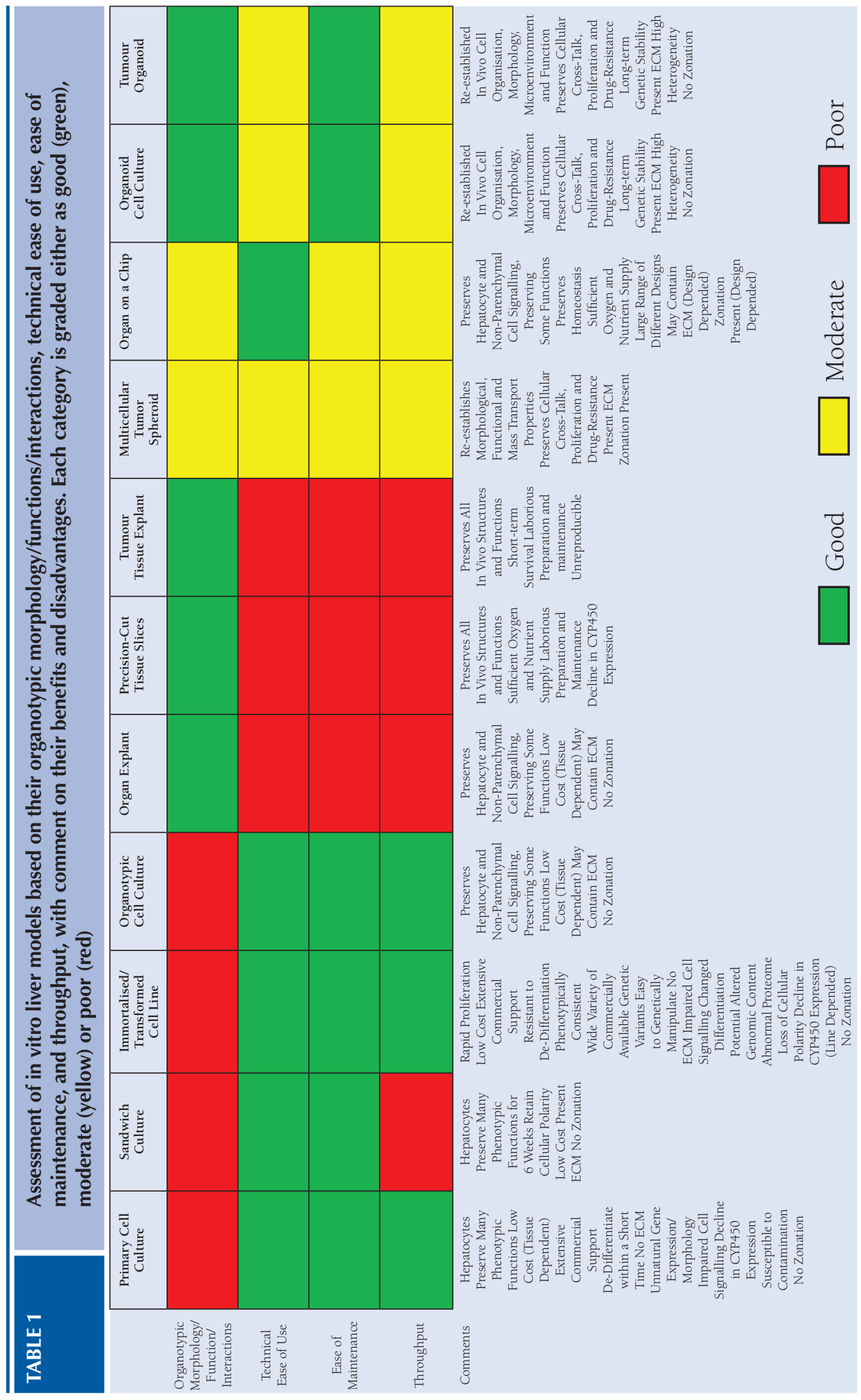


To date, there have only been a few successful clinical uses of in vitro organoid models. One example was a robust functional drug assay for cystic fibrosis, developed using human intestinal organoids, which demonstrated the clinical potential organoids hold for precision medicine (98). Using automated fluorescent image analysis, the function of the CFTR (which is defective in cystic fibrosis) can be assessed, allowing the authors to efficiently test drug responses of patients and treat rare forms of this disease (99). This assay has advantages over established in vitro models, such as rectal biopsies and primary airway tissue culture models because organoids can be passed into large screening arrays for high-throughput precision medicine (98).

\section{Tumor organoids}

Despite the precision medicine approach, only a minority of patients with cancer derive clear benefit from matching genetic targets with treatment. Currently, precision oncology based on emerging biomarkers remains an investigational strategy, and the present approach of matching single agents to patients is still suboptimal (17).

To address this issue, Pauli and colleagues piloted a study that combined whole-exome sequencing (WES) of patient metastatic and primary tumors with tumor organoid drug sensitivity assays, facilitating the integration of genomic data with drug screening in an iterative platform to identify effective therapeutic regimens for individual patients (100). Sequencing of 769 specimens identified somatic cancer gene alterations that were actionable by FDA-approved drugs in three specimens ( $0.4 \%$ of the total), but found somatic alterations with potential clinically actionable by off-label use in 71 of the remaining specimens $(9.6 \%$ of the total). Fifty-six organoid tumor lines and 19 patient-derived organoid xenografts were successfully established and characterized using cytology and histology, leading to patient-derived tumor organoids from four candidates being selected and subjected to $2 \mathrm{D}$ high-throughput drug screening. The tumors screened were from uterine carcinoma, endometrial carcinoma, and two lines of stage IV colorectal cancer. Single and combination compounds selected by this process were then validated in 3D cell culture. Drug combinations were further validated in patient-derived xenografts for two patients. In both cases, the drugs selected by the screening were found to be more effective at reducing tumor growth than the patient's current regimen. These results demonstrate that the optimal drug combinations can be identified using sequential drug-sensitivity screens followed by validation in personalized patient-derived tumor organoid xenograft models in a clinically relevant timeframe of 7 and 12 weeks (100). The further utility of tumor organoids to be passaged for large data sets while retaining individual phenotypic characteristics cannot be under-appreciated, as the power to rationally delineate the optimal therapy for every individual patient removes ambiguity and could exponentially speed up the rate of patient recovery. Pauli and colleagues demonstrated that 3D patient-derived tumor organoids can be a powerful tool for individual drug sensitivity assays, the results of which can be verified in xenograft models (100). However, these findings still need to be proven in clinical practice and shown to benefit patient outcomes. 
In late 2017, the clinical potential of tumor organoids derived from human primary liver cancer was demonstrated by Broutier and colleagues (101). Tumor organoids from HCC, cholangiocarcinoma (CC), and combined hepatocellularcholangiocarcinoma ( $\mathrm{CHC}$ ) retained features from their tissue of origin, such as the vast majority of cancer-related genetic variants, gene expression profiles, and tissue histologies. Immunohistochemistry and immunofluorescence showed that even after long-term expansion in culture, disease-specific protein expression was conserved, including the HCC markers HepParl and alpha-fetoprotein in HCC/ $\mathrm{CHC}$, and the ductal/CC marker epithelial cell adhesion molecule (EpCAM) in $\mathrm{CC} / \mathrm{CHC}$. Once established, the liver tumor organoid cultures were used to develop drug assays to identify patient-specific drug sensitivity. This was achieved by using a simple cell viability assay and observing the rate of organoid viability in the presence of range treatments with drugs such as sorafenib, gemcitabine, and SCH772984. Of these drugs, the sensitivity of the ERK inhibitor SCH772984 was then able to be validated in a patient-derived xenograft model transplanted with a CC tumor organoid (101).

Another precision medicine study by Nuciforo and colleagues, using human liver tumor organoids, found that HCC tumor-derived organoids maintained the growth pattern and differentiation grade of the originating primary tumor. In addition, alpha-fetoprotein, glypican 3, glutamine synthetase, and heat shock protein 70 protein expressions were preserved. Whole exome sequencing determined that somatic and non-synonymous somatic mutations in the HCC biopsies were observed at a rate of 88 and 90\%, respectively, in the corresponding HCC organoids at early passages. The tumor organoid cultures also displayed variable sensitivity to sorafenib exposure demonstrating that organoids derived from biopsies can be used to test tumor-specific sensitivities to growth-inhibitory substances. However, a direct comparison of in vitro sorafenib activity with the clinical response was not feasible, because none of the patients for whom organoid cultures were generated were treated with sorafenib (102).

These studies demonstrated the added value tumor organoids may have in the pursuit of precision medicine in treating primary liver cancer. While precision medicine has focused mainly on matching genetic targets with treatments, tumor organoids may be used to validate these matches in in vitro models or discover potential treatment options in the patient, which can be further validated in vivo, using tumor organoid xenograft models.

\section{FUTURE DIRECTIONS OF IN VITRO MODELS OF THE LIVER}

Future in vitro models of the liver need to be standardized to satisfy the requirements of (i) high-throughput with ease of use during cell maintenance and (ii) replication of anatomical and metabolic zonation of the liver lobule. Future in vitro models of the liver will combine material advancements made in organ-on-a-chip biotechnologies, bioprinting, and the cell biology advancements in organoid research. This could be achievable using permeable microfluidic tubes lined with LSECs to simulate blood flow and bile excretion or within a modified liver-on-achip system, populated by liver organoids and co-cultured with non-parenchymal cells, similar to the early intestinal organoid populated organ-on-a-chip devices 
recently developed, which have recapitulated important structural features and functions of the native duodenum $(103,104)$. Zonal inter-hepatic heterogeneity of the model may be controlled by applying an oxygen gradient across the hepatic cell population. Other considerations include assembly on a matrix that accurately models composition of the in vivo ECM for increased in vivo-like cell-ECM interactions. This would be similar to the bioprinted liver lobules created by Grix and colleagues, where populated HepaRG cells and human stellate cells had microchannel structures, which demonstrated flushing, higher levels of albumin, and CYP450 gene expression, while maintaining overall metabolism (105). The liver organoid-on-a-chip system by Wang and colleagues combined a perfusable organon-a-chip system with hiPSC-derived liver organoids, which demonstrated improved cell viability and higher expression of mature hepatic genes and endodermal genes (106).

\section{CONCLUSION}

In vitro models of liver disease represent an exciting opportunity to better understand liver homeostasis, response to injury, and cancer development. Conventional methods that use2D primary human hepatocytes and immortalized cell lines or 3D organ explant/PCTS have progressed to using 3D organ-on-a-chip and organoid models with microfluidics and appropriate co-cultures, in which the complex cellular heterogeneity of the originating organ is recapitulated ex vivo. Although well-characterized immortalized cell lines will remain relevant for studying highly conserved cellular processes and interactions, they cannot be regarded as completely accurate models of liver biology in vivo. It is also possible that in the future, as methods become established and validated, in vitro models of the liver will increase the efficacy of pre-clinical drug development, leading to more therapies to treat liver disease. Tumor-derived organoids may also play an essential role in fulfilling the promises of precision medicine, as a method of validating prospective drugs for individual patients.

Conflict of Interest: The authors declare no potential conflict of interest with respect to research, authorship, and/or publication of this manuscript.

Copyright and Permission Statement: To the best of our knowledge, the materials included in this chapter do not violate copyright laws. All original sources have been appropriately acknowledged and/or referenced. Where relevant, appropriate permissions have been obtained from the original copyright holder(s).

\section{REFERENCES}

1. Astashkina A, Mann B, Grainger DW. A critical evaluation of in vitro cell culture models for highthroughput drug screening and toxicity. Pharmacol Ther. 2012;134(1):82-106. http://dx.doi. org/10.1016/j.pharmthera.2012.01.001

2. Zeilinger K, Freyer N, Damm G, Seehofer D, Knöspel F. Cell sources for in vitro human liver cell culture models. Exp Biol Med (Maywood). 2016;241(15):1684-98. http://dx.doi.org/10.1177/ 1535370216657448 
3. Lu FC. Biotransformation of toxicants. In: Basic toxicology: Fundamentals, target organs and risk assessment. 3rd ed. Washington, DC: Taylor and Francis; 1996. p. 27-39.

4. Kietzmann T. Metabolic zonation of the liver: The oxygen gradient revisited. Redox Biol. 2017;11: 622-30. http://dx.doi.org/10.1016/j.redox.2017.01.012

5. Jungermann K, Kietzmann T. Oxygen: Modulator of metabolic zonation and disease of the liver. Hepatology. 2000;31:255-60. http://dx.doi.org/10.1002/hep.510310201

6. Soto-Gutierrez A, Gough A, Vernetti LA, Taylor DL, Monga SP. Pre-clinical and clinical investigations of metabolic zonation in liver diseases: The potential of microphysiology systems. Exp Biol Med. 2017;242(16):1605-16. http://dx.doi.org/10.1177/1535370217707731

7. Moffat JG, Vincent F, Lee JA, Eder J, Prunotto M. Opportunities and challenges in phenotypic drug discovery: An industry perspective. Nat Rev Drug Discov. 2017;16(8):531-43. http://dx.doi. org/10.1038/nrd.2017.111

8. Avorn J. The $\$ 2.6$ billion pill-Methodologic and policy considerations. N Engl J Med. 2015; 372(20):1877-9. http://dx.doi.org/10.1056/NEJMp1500848

9. Di Masi JA, Grabowski HG, Hansen RW. The cost of drug development. N Engl J Med. 2015;372:1972. http://dx.doi.org/10.1056/NEJMc1504317

10. Zhang D, Luo G, Ding X, Lu C. Preclinical experimental models of drug metabolism and disposition in drug discovery and development. Acta Pharmaceutica Sinica B. 2012;2(6):549-61. http://dx.doi. org/10.1016/j.apsb.2012.10.004

11. Tsaioun K, Jacewicz M. De-Risking Drug discovery with ADDME—Avoiding drug development mistakes early. Altern Lab Anim. 2009;37(Suppl 1):47-55. http://dx.doi.org/10.1177/026119290903701S10

12. Xu JJ, Diaz D, O'Brien PJ. Applications of cytotoxicity assays and pre-lethal mechanistic assays for assessment of human hepatotoxicity potential. Chem Biol Interact. 2004;150(1):115-28. http:// dx.doi.org/10.1016/j.cbi.2004.09.011

13. Lee WM. Drug-induced hepatotoxicity. N Engl J Med. 2003;349(5):474-85. http://dx.doi. org/10.1056/NEJMra021844

14. Collins FS, MacKusick VA. Implications of the human genome project for medical science. JAMA. 2001;285(5):540-4. http://dx.doi.org/10.1001/jama.285.5.540

15. Ginsburg GS, Phillips KA. Precision medicine: From science to value. Health Aff (Millwood). 2018;37(5):694-701. http://dx.doi.org/10.1377/hlthaff.2017.1624

16. Ashley EA. Towards precision medicine. Nat Rev Genet. 2016;17:507-22. http://dx.doi.org/10.1038/ nrg.2016.86

17. Remon J, Dienstmann R. Precision oncology: Separating the wheat from the chaff. ESMO Open. 2018;3(6):e000446. http://dx.doi.org/10.1136/esmoopen-2018-000446

18. Ponsoda X, Pareja E, Gómez-Lechón M, Fabra R, Carrasco E, Trullenque R, et al. Drug biotransformation by human hepatocytes. In vitro/in vivo metabolism by cells from the same donor. J Hepatol. 2001;34(1):19-25. http://dx.doi.org/10.1016/S0168-8278(00)00085-4

19. Gómez-Lechón MJ, Donato MT, Castell JV, Jover R. Human hepatocytes as a tool for studying toxicity and drug metabolism. Curr Drug Metab. 2003;4(4):292-312. http://dx.doi.org/10.2174/ 1389200033489424

20. Caron JM. Induction of albumin gene transcription in hepatocytes by extracellular matrix proteins. Mol Cell Biol. 1990;10(3):1239-43. http://dx.doi.org/10.1128/MCB.10.3.1239

21. Lee YJ, Streuli CH. Extracellular matrix selectively modulates the response of mammary epithelial cells to different soluble signaling ligands. J Biol Chem. 1999;274(32):22401-8. http://dx.doi. org/10.1074/jbc.274.32.22401

22. Page JL, Johnson MC, Olsavsky KM, Strom SC, Zarbl H, Omiecinski CJ. Gene expression profiling of extracellular matrix as an effector of human hepatocyte phenotype in primary cell culture. Toxicol Sci. 2007;97(2):384-97. http://dx.doi.org/10.1093/toxsci/kfm034

23. Michalopoulos G, Cianciulli HD, Novotny AR, Kligerman AD, Strom SC, Jirtle RL. Liver regeneration studies with rat hepatocytes in primary culture. Cancer Res. 1982;42(11):4673-82.

24. Ben-Ze'ev A, Robinson GS, Bucher NL, Farmer SR. Cell-cell and cell-matrix interactions differentially regulate the expression of hepatic and cytoskeletal genes in primary cultures of rat hepatocytes. Cell Biol. 1988;85:2161-5. http://dx.doi.org/10.1073/pnas.85.7.2161 
25. Bataller R, Brenner DA. Liver fibrosis. J Clin Investig. 2005;115(2):209-18. http://dx.doi.org/10.1172/ JCI24282

26. Wasser S, Tan C E. Experimental models of hepatic fibrosis in the rat. Ann Acad Med Singapore. 1999;28(1):109-11.

27. Kegel V, Deharde D, Pfeiffer E, Zeilinger K, Seehofer D, Damm G. Protocol for isolation of primary human hepatocytes and corresponding major populations of non-parenchymal liver cells. J Vis Exp. 2016;109:e53069. http://dx.doi.org/10.3791/53069

28. Gómez-Lechón MJ, Castell JV, Donato MT. Hepatocytes-The choice to investigate drug metabolism and toxicity in man: In vitro variability as a reflection of in vivo. Chem Biol Interact. 2007;168(1): 30-50. http://dx.doi.org/10.3791/53069

29. Ranga A, Gjorevski N, Lutolf MP. Drug discovery through stem cell-based organoid models. Adv Drug Deliv Rev. 2014;69-70:19-28. http://dx.doi.org/10.1016/j.addr.2014.02.006

30. Hartung T, Daston G. Are in vitro tests suitable for regulatory use? Toxicol Sci. 2009;111(2):233-7. http://dx.doi.org/10.1093/toxsci/kfpl49

31. Wright MC, Paine AJ. Evidence that the loss of rat liver cytochrome P450 in vitro is not solely associated with the use of collagenase, the loss of cell-cell contacts and/or the absence of an extracellular matrix. Biochem Pharmacol. 1992;43(2):237-43. http://dx.doi.org/10.1016/0006-2952(92)90283-O

32. Rodríguez-Antona C, Donato MT, Boobis A, Edwards RJ, Watts PS, Vicente Castell J, et al. Cytochrome P450 expression in human hepatocytes and hepatoma cell lines: Molecular mechanisms that determine lower expression in cultured cells. Xenobiotica. 2002;32(6):505-20. http://dx.doi. org/10.1080/00498250210128675

33. Le Cluyse EL, Audus KL, Hochman JH. Formation of extensive canalicular networks by rat hepatocytes cultured in collagen-sandwich configuration. Am J Physiol. 1994;266(6 Pt 1):C1764-74. http:// dx.doi.org/10.1152/ajpcell.1994.266.6.C1764

34. Liu X, Brouwer KL, Gan LS, Brouwer KR, Stieger B, Meier PJ, et al. Partial maintenance of taurocholate uptake by adult rat hepatocytes cultured in a collagen sandwich configuration. Pharm Res. 1998;15(10):1533-9. http://dx.doi.org/10.1023/A:1011994831139

35. Dunn JCY, Tompkins RG, Yarmush ML. Long-term in vitro function of adult hepatocytes in a collagen sandwich configuration. Biotechnol Prog. 1991;7(3):237-45. http://dx.doi.org/10.1021/ bp00009a007

36. Dunn JCY, Tompkins RG, Yarmush ML. Hepatocytes in collagen sandwich: Evidence for transcriptional and translational regulation. J Cell Biol. 1992;116(4):1043-53. http://dx.doi.org/10.1083/ jcb.116.4.1043

37. Kern A, Bader A, Pichlmayr R, Sewing KF. Drug metabolism in hepatocyte sandwich cultures of rats and humans. Biochem Pharmacol. 1997;54:761-72. http://dx.doi.org/10.1016/S0006-2952(97)00204-9

38. Yanni SB, Augustijns PF, Benjamin DK, Brouwer KLR, Thakker DR. Annaert PP. In vitro investigation of the hepatobiliary disposition mechanisms of the antifungal agent micafungin in humans and rats. Drug Metab Dispos. 2010;38(10):1848-56. http://dx.doi.org/10.1124/dmd.110.033811

39. Yang K, Guo C, Woodhead JL, St. Claire RL, Watkins PB, Siler SQ, et al. Sandwich-cultured hepatocytes as a tool to study drug disposition and drug-induced liver injury. J Pharm Sci. 2016;105: 443-59. http://dx.doi.org/10.1016/j.xphs.2015.11.008

40. Gripon P, Rumin S, Urban S, Le Seyec J, Glaise D, Cannie I, et al. Infection of a human hepatoma cell line by hepatitis B virus. Proc Natl Acad Sci U S A. 2002;99(24):15655-60. http://dx.doi.org/10.1073/ pnas. 232137699

41. Verrier ER, Colpitts CC, Schuster C, Zeisel MB, Baumert TF. Cell culture models for the investigation of hepatitis B and D virus infection. Viruses. 2016;8(9):261. http://dx.doi.org/10.3390/v8090261

42. Lempp F, Schlund F, Rieble L, Nussbaum L, Eck C, Ni Y, Urban S. SAT-373—Recapitulation of the complete HDV replication cycle in a novel hepatoma cell line allows for efficient antiviral compound evaluation. J Hepatol. 2018;68(1):S775. http://dx.doi.org/10.1016/S0168-8278(18)31818-X

43. Verrier ER, Colpitts CC, Bach C, Heydmann L, Weiss A, Renaud M, et al. A targeted functional RNA interference screen uncovers glypican 5 as an entry factor for hepatitis B and D viruses. Hepatology. 2016;63(1):35-48. http://dx.doi.org/10.1002/hep.28013 
44. Yamasaki K, Kawasaki S, Young RD, Fukuoka H, Tanioka H, Nakatsukasa M, et al. Genomic aberrations and cellular heterogeneity in SV40-immortalized human corneal epithelial cells. Invest Ophthalmol Vis Sci. 2009;50:604-13. http://dx.doi.org/10.1167/iovs.08-2239

45. Prozialeck WC, Edwards JR, Lamar PC, Smith CS. Epithelial barrier characteristics and expression of cell adhesion molecules in proximal tubule-derived cell lines commonly used for in vitro toxicity studies. Toxicol In Vitro. 2006;20(6):942-53. http://dx.doi.org/10.1016/j.tiv.2005.11.006

46. Milyavsky M, Shats I, Erez N, Tang X, Senderovich S, Meerson A, et al. Prolonged culture of telomerase-immortalized human fibroblasts leads to a premalignant phenotype. Cancer Res. 2003;63: 7147-57. http://dx.doi.org/10.1016/j.biomaterials.2010.07.101

47. Holt DJ, Chamberlin LM, Grainger DW. Cell-cell signaling in co-cultures of macrophages and fibroblasts. Biomaterials. 2010;31(36):9382-94. http://dx.doi.org/10.2174/138920008783331086

48. Cummings BS, Lasker JM, Lash LH. Expression of glutathione-dependent enzymes and cytochrome P450s in freshly isolated and primary cultures of proximal tubular cells from human kidney. J Pharmacol Exp Ther. 2000;293:677-85.

49. Donato MT, Lahoz A, Castell JV, Gómez-Lechón MJ. Cell lines: A tool for in vitro drug metabolism studies. Curr Drug Metab. 2008;9(1):1-11. http://dx.doi.org/10.2174/138920008783331086

50. Chamberlain LM, Godek ML, Gonzalez-Juarrero M, Grainger DW. Phenotypic non-equivalence of murine (monocyte-) macrophage cells in biomaterial and inflammatory models. J Biomed Mater Res A. 2009;88(4):858-71. http://dx.doi.org/10.1002/jbm.a.31930

51. Pan C, Kumar C, Bohl S, Klingmueller U, Mann M. Comparative proteomic phenotyping of xell lines and primary cells to assess preservation of cell type-specific functions. Mol Cell Proteomics. 2009;8(3):443-50. http://dx.doi.org/10.1074/mcp.M800258-MCP200

52. Ramboer E, Vanhaecke T, Rogiers V, Vinken M. Immortalized human hepatic cell lines for in vitro testing and research purposes. Methods Mol Biol. 2015;1250:53-76. http://dx.dor. org/10.1007/978-1-4939-2074-7_4

53. Kato N, Ikeda M, Mizutani T, Sugiyama K, Noguchi M, Hirohashi S, et al. Replication of hepatitis C virus in cultured non-neoplastic human hepatocytes. Jpn J Cancer Res. 1996;87:787-92. http:// dx.doi.org/10.1111/j.1349-7006.1996. tb02101.x.

54. Ikeda M, Kato N, Mizutani T, Sugiyama K, Tanaka K, Shimotohno K. Analysis of the cell tropism of HCV by using in vitro HCV infected human lymphocytes and hepatocytes. J Hepatol. 1997;27:445-54. http://dx.doi.org/10.1016/S0168- 8278(97)80347-9.

55. Brown JJ, Parashar B, Moshage H, Tanaka KE, Engelhardt D, Rabbani E, et al. A long-term hepatitis B viremia model generated by transplanting non-tumorigenic immortalized human hepatocytes in Rag-2-deficient mice. Hepatology. 2000;31:173-81. http://dx.doi.org/10.1002/ hep. 510310126.

56. Yu S, Chen J, Wu M, Chen H, Kato N, Yuan Z. Hepatitis B virus polymerase inhibits RIG-I- and Tolllike receptor 3-mediated beta interferon induction in human hepatocytes through interference with interferon regulatory factor 3 activation and dampening of the interaction between TBK1/IKK epsilon and DDX3. J Gen Virol. 2010;91:2080-90. http://dx.doi.org/10.1099/vir.0.020552-0.

57. Heim D, Cornils K, Schulze K, Fehse B, Lohse AW, Brümmendorf TH, et al. Retroviral insertional mutagenesis in telomerase-immortalized hepatocytes identifies RIPK4 as novel tumor suppressor in human hepatocarcinogenesis. Oncogene. 2015; 34: 364-372. http://dx.doi.org/10.1038/ onc.2013.551

58. De Gottardi A, Vinciguerra M, Sgroi A, Moukil M, Ravier-Dall'Antonia F, Pazienza V, et al. Microarray analyses and molecular profiling of steatosis induction in immortalized human hepatocytes. Lab Invest. 2007;87:792-806. http://dx.doi.org/10.1038/labinvest.3700590.

59. Waki K, Anno K, Ono T, Ide T, Chayama K, Tahara H. Establishment of functional telomerase immortalized human hepatocytes and a hepatic stellate cell line for telomere-targeting anticancer drug development. Cancer Sci. 2010;101(7):1678-85. http://dx.doi.org/10.1111/j.1349-7006.2010.01576.x

60. Godoy P, Hewitt NJ, Albrecht U, Andersen ME, Ansari N, Bhattacharya S, et al. Recent advances in $2 \mathrm{D}$ and $3 \mathrm{D}$ in vitro systems using primary hepatocytes, alternative hepatocyte sources and nonparenchymal liver cells and their use in investigating mechanisms of hepatotoxicity, cell signaling and ADME. Arch Toxicol. 2013;87(8):1315-530. http://dx.doi.org/10.1007/s00204-013-1078-5 
61. Bale SS, Golberg I, Jindal R, McCarty WJ, Luitje M, Hegde M, et al. Long-term coculture strategies for primary hepatocytes and liver sinusoidal endothelial cells. Tissue Eng Part C Methods. 2015;21(4):413-22. http://dx.doi.org/10.1089/ten.tec.2014.0152

62. Kidambi S, Yarmush RS, Novik E, Chao P, Yarmush ML, Nahmias Y. Oxygen-mediated enhancement of primary hepatocyte metabolism, functional polarization, gene expression, and drug clearance. Proc Natl Acad Sci U S A. 2009;106(37):15714-19. http://dx.doi.org/10.1073/pnas.0906820106

63. Ware BR, Durham MJ, Monckton CP, Khetani SR. A cell culture platform to maintain long-term phenotype of primary human hepatocytes and endothelial cells. Cellular Mol Geastroenterol Hepatol. 2018;5(3):187-207. http://dx.doi.org/10.1016/j.jcmgh.2017.11.007

64. Mitra A, Mishra L, Li S. Technologies for deriving primary tumor cells for use in personalized cancer therapy. Trends Biotechnol. 2013;31(6):347-54. http://dx.doi.org/10.1016/j.tibtech.2013.03.006

65. Torricelli P, Antonelli F, Ferorelli P, De Martino A, Shevchenko A, Siciliano A, et al. Organ culture model of liver for the study of cancer treatment for hepatocellular carcinoma. Cancer Res J. 2016;4(2):37-42. http://dx.doi.org/10.11648/j.crj.20160402.13

66. de Graaf IAM, Olinga P, de Jager MH, Merema MT, de Kanter R, van de Kerkhof EG, et al. Preparation and incubation of precision-cut liver and intestinal slices for application in drug metabolism and toxicity studies. Nat Protocols. 2010;5(9):1540-51. http://dx.doi.org/10.1038/nprot.2010.111

67. Groothuis GM, Hulstaert CE, Kalicharan D, Hardonk MJ. Plasma membrane specialization and intracellular polarity of freshly isolated rat hepatocytes. Eur J Cell Biol. 1981;26(1):43-51.

68. Vickers AE, Saulnier M, Cruz E, Merema MT, Rose K, Bentley P, et al. Organ slice viability extended for pathway characterization: An in vitro model to investigate fibrosis. Toxicol Sci. 2004;82(2):534-44. http://dx.doi.org/10.1093/toxsci/kfh285

69. de Graaf IAM, Groothuis GMM, Olinga P. Precision-cut tissue slices as a tool to predict metabolism of novel drugs. Expert OpinDrug Metab Toxicol. 2007;3(6):879-98. http://dx.doi.org/10.1517/ 17425255.3.6.879

70. Freeman AE, Hoffman RM. In vivo-like growth of human tumors in vitro. Proc Natl Acad Sci U S A. 1986;83(8):2694-8. http://dx.doi.org/10.1073/pnas.83.8.2694

71. Vaira V, Fedele G, Pyne S, Fasoli E, Zadra G, Bailey D, et al. Preclinical model of organotypic culture for pharmacodynamic profiling of human tumors. Proc Natl Acad Sci U S A. 2010;107(18):8352-6. http://dx.doi.org/10.1073/pnas.0907676107

72. Nath S, Devi GR. Three-dimensional culture systems in cancer research: Focus on tumor spheroid model. Pharmacol Ther. 2016;163:94-108. http://dx.doi.org/10.1016/j.pharmthera.2016.03.013

73. Weiwald LB, Bellet D, Dangles-Marie V. Spherical cancer models in tumor biology. Neoplasia. 2015;17(1):1-15. http://dx.doi.org/10.1016/j.neo.2014.12.004

74. Friedrich J, Seidel C, Edner R, Kanz-Schughart LA. Spheroid-based drug screen: Considerations and practical approach. Nat Protoc. 2009;4(3):309-24. http://dx.doi.org/10.1038/nprot.2008.226

75. Song Y, Kim S, Kim KM, Choi EK, Kim J, Seo HR. Activated hepatic stellate cells play pivotal roles in hepatocellular carcinoma cell chemoresistance and migration in multicellular tumor spheroids. Sci Rep. 2016;6:36750. http://dx.doi.org/10.1038/srep36750

76. Seo HR. Roles of tumor microenvironment in hepatocelluar carcinoma. Curr Cancer Ther Rev. 2015;11:82-93. http://dx.doi.org/10.2174/1573394711666151022203313

77. Jung HR, Kang HM, Ryu JW, Kim DS, Noh KH, Kim ES, et al. Cell spheroids with enhanced aggressiveness to mimic human liver cancer in vitro and in vivo. Sci Rep. 2017;7:10499. http://dx.doi. org/10.1038/s41598-017-10828-7

78. Esch EW, Bahinski A, Huh D. Organs-on-chips at the frontiers of drug discovery. Nat Rev Drug Discov. 2015;14(4):248-60. http://dx.doi.org/10.1038/nrd4539

79. Usta OB, McCarty WJ, Bale S, Hegde M, Jindal R, Bhushan A, et al. Microengineered cell and tissue systems for drug screening and toxicology applications: Evolution of in-vitro liver technologies. Technology (Singap World Sci). 2015;3(1):1-26. http://dx.doi.org/10.1142/S2339547815300012

80. Starokozhko V, Groothuis GM. Judging the value of 'liver-on-a-chip' devices for prediction of toxicity. Expert Opin Drug Metab Toxicol. 2017;13(2):125-8. http://dx.doi.org/10.1080/17425255.2017.124 6537 
81. Snouber LC, Bunescu A, Naudot M, Legallais C, Brochot C, Dumas ME, et al. Metabolomics-on-achip of epatotoxicity induced by anticancer drug flutamide and its active metabolite hydroxyflutamide using HepG2/C3a microfluidic biochips. Toxicol Sci. 2013;132(1):8-20. http://dx.doi.org/10.1093/ toxsci/kfs230

82. Bhise NS, Manoharan V, Massa S, Tamayol A, Ghaderi M, Miscuglio M, et al. A liver-on-a-chip platform with bioprinted hepatic spheroids. Biofabrication. 2016;8(1):014101. http://dx.doi.org/10.1088/ 1758-5090/8/1/014101

83. Baudoin R, Prot JM, Nicolas G, Brocheton J, Brochot C, Legallais C, et al. Evaluation of seven drug metabolisms and clearances by cryopreserved human primary hepatocytes cultivated in microfluidic biochips. Xenobiotica. 2013;43(2):140-52. http://dx.doi.org/10.1088/1758-5090/8/1/014101

84. Yu Y, Liu H, Ikeda Y, Amiot BP, Rinaldo P, Duncan SA, Nyberg SL. Hepatocyte-like cells differentiated from human induced pluripotent stem cells: Relevance to cellular therapies. Stem Cell Res. 2012;9(3):196-207. http://dx.doi.org/10.1016/j.scr.2012.06.004

85. Allen JW, Bhatia SN. Formation of steady-state oxygen gradients in vitro application to liver zonation. Biotechnol Bioeng. 2003;82(3):253-62. http://dx.doi.org/10.1002/bit. 10569

86. Allen JW, Khetani SR, Bhatia SN. In vitro zonation and toxicity in a hepatocyte bioreactor. Toxicol Sci. 2005;84:110-19. http://dx.doi.org/10.1093/toxsci/kfi052

87. Lancaster MA, Knoblich JA. Organogenesis in a dish: Modeling development and disease using organoid technologies. Science. 2014;345(6194):1247125. http://dx.doi.org/10.1126/science.1247125

88. Clevers H. Modeling development and disease with organoids. Cell. 2016;165(7):1586-97. http:// dx.doi.org/10.1016/j.cell.2016.05.082

89. Huch M, Gehart H, van Boxtel R, Hamer K, Blokzijl F, Verstegen MMA, et al. Long-term culture of genome-stable bipotent stem cells from adult human liver. Cell. 2015;160(1-2):299-312. http:// dx.doi.org/10.1016/j.cell.2014.11.050

90. Huch M, Dorrell C, Boj SF, van Es JH, Li VSW, van de Wetering M, et al. In vitro expansion of single Lgr5+ liver stem cells. Nature. 2013;494(7436):247-50. http://dx.doi.org/10.1038/naturel 1826

91. Nantasanti S, de Bruin A, Rothuizen J, Penning LC, Schotanus BA. Concise review: Organoids are a powerful tool for the study of liver disease and personalized treatment design in humans and animals. Stem Cells Transl Med. 2016;5(3):325-30. http://dx.doi.org/10.5966/sctm.2015-0152

92. Broutier L, Anderson-Rolf A, Hindley CJ, Boj SF, Clevers H, Koo B, et al. Culture and establishment of self-renewing human and mouse adult liver and pancreas 3D organoids and their genetic manipulation. Nat Protoc. 2016;11(9):1724-43. http://dx.doi.org/10.1038/nprot.2016.097

93. Schwank G, Koo BK, Sasselli V, Dekkers JF, Heo I, Demircan T, et al. Functional repair of CFTR by CRISPR/Cas9 in intestinal stem cell organoids of cystic fibrosis patients. Cell Stem Cell. 2013;13(6):653-8. http://dx.doi.org/10.1016/j.stem.2013.11.002

94. Lee JS, Shin J, Park H, Kim Y, Kim B, Oh J, et al. Liver extracellular matrix providing dual functions of two-dimensional substrate coating and three-dimensional injectable hydrogel platform for liver tissue engineering. Biomacromolecules. 2014;15(1):206-18. http://dx.doi.org/10.1021/bm4015039

95. Fatehullah A, Tan SH, Barker N. Organoids as an in vitro model of human development and disease. Nat Cell Biol. 2016;18(3):246-54. http://dx.doi.org/10.1038/ncb3312

96. Huch M, Knoblich JA, Lutolf MP, Martinez-Arias A. The hope and the hype of organoid research. Development. 2017;144:938-41. http://dx.doi.org/10.1242/dev.150201

97. Astashkina A, Grainger DW. Critical analysis of 3-D organoid in vitro cell culture models for highthroughput drug candidate toxicity assessments. Adv Drug Deliv Rev. 2014;69-70:1-18. http:// dx.doi.org/10.1016/j.addr.2014.02.008

98. Dekkers JF, Wiegerinck CL, de Jonge HR, Bronsveld I, Janssens HM, de Winter-de Groot KM, et al. A functional CFTR assay using primary cystic fibrosis intestinal organoids. Nat Med. 2013;19(7): 939-45. http://dx.doi.org/10.1038/nm.3201

99. Chakradhar S. Put to the test: Organoid-based testing becomes a clinical tool. Nat Med. 2017;23(7):796-9. http://dx.doi.org/10.1038/nm0717-796

100. Pauli C, Hopkins BD, Prandi D, Shaw R, Fedrizzi T, Sboner A, et al. Personalized in vitro and in vivo cancer models to guide precision medicine. Cancer Discov. 2017;7(5):462-77. http://dx.doi. org/10.1158/2159-8290.CD-16-1154 
101. Broutier L, Mastrogiovanni G, Verstegen MMA, Francies HE, Gavarró LM, Bradshaw CR, Allen GE, et al. Human primary liver cancer-derived organoid cultures for disease modeling and drug screening. Nat Med. 2017;23:1424-35. http://dx.doi.org/10.1038/nm.4438

102. Nuciforo S, Fofana I, Matter MS, Blumer T, Calabrese D, Boldanova T, et al. Organoid models of human liver cancers derived from tumor needle biopsies. Cell Rep. 2018;24(5):1363-76. http:// dx.doi.org/10.1016/j.celrep.2018.07.001

103. Workman MJ, Gleeson JP, Troisi EJ, Estrada HQ, Kerns SJ, Hinojosa CD, et al. Enhanced utilization of induced pluripotent stem cell-derived human intestinal organoids using microengineered chips. Cell Mol Gastroenterol Hepatol. 2017;5(4):669-77.e2. http://dx.doi.org/10.1016/j.jcmgh.2017.12.008

104. Kasendra M, Tovaglieri A, Sontheimer-Phelps A, Jalili-Firoozinezhad S, Bein A, Chalkiadakil A, et al. Development of a primary human small intestine-on-a-chip using biopsy-derived organoids. Sci Rep. 2018;8: 2871. http://dx.doi.org/10.1038/s41598-018-21201-7

105. Grix T, Ruppelt A, Thomas A, Amler A, Noichl BP, Lauster R, et al. Bioprinting perfusion-enabled liver equivalents for advanced organ-on-a-chip applications. Genes. 2018;9:176. http://dx.doi. org/10.3390/genes9040176

106. Wang Y, Wang H, Deng P, Chen W, Guo Y, Tao T, et al. In situ differentiation and generation of functional liver organoids from human iPSCs in a 3D perfusable chip system. Lab Chip. 2018;18:3606. http://dx.doi.org/10.1039/C8LC00869H 